\title{
Language disorders in preschool Egyptian children with stuttering
}

\author{
Original \\ Effat Ahmed zaky, Haytham Mamdouh, Marowa Abd El Wahab, Shima Osman, \\ Article \\ Zeinab Khalaf
}

Department of Otolaryngology and Unit of Phoniatrics, Faculty of Medicine, Minia University, Egypt.

\begin{abstract}
Background /Aims: Stuttering typically emerges in the preschool years as children are experiencing substantial growth in their language and are beginning to combine words to form short sentences. Stuttering occurs during the process of planning utterances and using sounds to create words and sentences. A significant percentage of stutterers had a history of delayed language development and articulation disorders. This study's purpose was to examine differences between children who stutter (CWS) and child who do not stutter (CWNS) on standardized tests of IQ, language test, and articulatory tests.

Patients and Methods: An analytic cross sectional study conducted on two groups of subjects. The study group consisted of 52 children (study group) who stutter (CWS) and control group included 52 children who do not stutter (CWNS). Each child in the study group was audio-recorded, which was subsequently assessed by (Arabic version of stuttering severity index "A-SSI") to provide information pertinent to the child's frequency and type of speech dysfluencies. Each child was determined and responded to standardized tests of cognitive, language, and articulatory assessment.

Results: There was a significant decrease in receptive, expressive, and total language scores of language test within cases; regards to articulation test, there was a considerable increase of SSDs. These differences in receptive/expressive language and total language scores were significantly correlated with the overall stuttering frequency of cases CWS.

Conclusion: Findings were taken to suggest the imbalance among components of the speech-language systems of CWS that may contribute to the difficulties they have establishing usual speech fluency.
\end{abstract}

Key Words: Language, preschool, stuttering.

Received: 14 November 2020, Accepted: 3 February 2021

Corresponding Author: Effat Ahmed zaky, MD, Department of Otolaryngology and Unit of Phoniatrics, Faculty of Medicine, Minia University, Tel.: 0020-01016607002, E-mail: dr_efat_am@yahoo.com

ISSN: 2090-0740, 2021

\section{INTRODUCTION}

In the profession of communication disorders, it is commonly reported that children who stutter, as a group, are more likely to have phonological and language disorders than their non-stuttering peers. Some support for this belief comes from survey studies that have questioned phoniatricians about the children on their caseloads who stutter. Recently, one such study reported that $44 \%$ of children who stuttered had at least one additional communication disorder ${ }^{[1]}$. Fluency is the production of a more or less continuous speech at a relatively rapid rate with optimum effort ${ }^{[2]}$. Many variables determine fluency reflecting the temporal aspects of speech production as pauses, rhythm, intonation, stresses, and rate of speech ${ }^{[3]}$. Stuttering is a multifactorial speech disorder defined by frequent prolongations, repetitions, or blocks of spoken sounds and/or syllables. It is a common disorder affecting about $5 \%$ of children; only $20 \%$ of them continue to stutter ${ }^{[4]}$ to become $1 \%$ of adults ${ }^{[5]}$. There are at least four significant explanations for fluency failure. One type of dysfluent speech is the result of a normal developmental process, and the remaining four are the result of different abnormalities. A child may be dysfluent due to passing through the necessary or stressful stages of language learning. These developmental dysfluencies were considered as normal part of the acquisition of our language. A person may be dysfluent as a result of a neurogenic dysfunction, which may affect communication. Some dysfluencies are psychogenic, and some dysfluencies are the result of delay language developmental ${ }^{[6]}$. Stuttering typically emerges in the pre-school years as children are experiencing substantial growth in their language and are beginning to combine words to form short sentences ${ }^{[7]}$. Stuttering occurs during the process of planning utterances and selecting and using sounds to create words and sentences ${ }^{[8]}$. Arndt and Healey ${ }^{[9]}$ conducted a study to determine the number of children stuttered with co-occurring language disorders and phonological disorders. They concluded, based on the survey data from 241 speech-language pathologists (SLPs) from 10 states in the United States, that $56 \%$ of the 467 children who stuttered had verified fluency disorders only. 
In contrast, 44\% (205) had verified fluency and concomitant phonological and/or language disorders. A survey of 190 stutterers was obtained and analyzed to determine the incidence of other speech, language, and learning problems for stutterers and family members. Results indicated that a significant percentage of their sample of stutterers had a history of delayed language development and articulation disorders. A smaller percentage reported learning and written language problems. So significant relationships were discovered between stuttering, articulation disorders, delayed language, and written language problems ${ }^{[10]}$.

This work aimed at establishing baseline data about the size and distribution of language disorders among Egyptian stuttering children to put a plan of early detection, proper assessment and intervention of these problems if possible.

\section{PATIENTS AND METHODS:}

\section{Subjects:}

An analytic cross sectional study conducted on two groups of subjects. The study group consisted of 52 children (study group) who stutter (CWS) and control group included 52 children who do not stutter (CWNS). The study group: This group included 52 children with stuttering. They were 43 males $(42.7 \%)$ and 9 females $(17.3 \%)$ with a range of age 3 years to 7 years. The subjectinclusion criteria for the study group: preschool aged children, children whose main complaints are stuttering disorders and children who stutter at least six months diagnosed by expert phoniatricians. The studied children were native speakers of Egyptian Arabic with no hearing, neurological, developmental, intellectual or emotional problems based on parental report and examination). While, the exclusion criteria in this study were schooling age children, non-stuttered children and children referred for remediation of language and /or articulation disorder in conjunction with stuttering. The control group (CWNS): This group included 52 children without stuttering. They were 39 males $(75 \%)$ and 13 females $(25 \%)$, with a range of age 2 years to 7 years. Patients were selected from outpatient clinic of Phoniatrics, Minia University Hospital (study group) and ENT clinic (control group) of Minia University Hospitals.

\section{Methods:}

All children were assessed according to the assessment protocol in the Phoniatrics clinics, Minia University Hospital. This protocol was classified into:

\section{A-Preliminary Diagnostic Procedure:}

1-Parents interview and history including complaint, personal data, searching for etiological factors during pregnancy, natal, neonatal and postnatal periods, developmental milestones and illness of early childhood, it also included detailed analysis of stuttering history.

2-Examination including neurological and ENT including ear, nose and throat examination. 3-Subjective auditory perceptual assessment "APA" of both language and speech (automatic and spontaneous speech) during child-parent $\backslash$ physician conversation.

\section{B-Clinical Diagnostic Aids:}

(1)- Cognitive functions assessment by Stanford Binet intelligence scale fifth edition: The Arabic version of Stanford Binet test fifth edition for assessment of intelligence was used ${ }^{[10]}$.

(2)- Language test by Arabic Preschool Language Scale-4 "APLS-4"[11]. This modified scale was used mainly to identify children who had a language disorder or delay.

(3)- Mansoura Arabic Articulatory Test "MAAT": A detailed assessment of articulation skills was carried out in the phoniatrics clinics of Mansoura University with the aid of systematic articulation test that covered all Arabic sounds ${ }^{[12]}$.

(4)- Documentation of auditory perceptual assessment recording using Sony Xperia Z2 casseta. The speech recording was carried in a sound treated room with a minimal noise level and fixed distance" $15 \mathrm{~cm}$ " between The speaker and The recorder. The recorded samples were used for calculation of the number of dysfluencies within the speech sample estimation of percentage of dysfluent speech according to the following equation: $\mathrm{SS} \%=$ Number of stuttered words $\backslash$ Number of fluent and dysfluent $\times 100$.

(5)- Arabic version of stuttering severity index "A-SSI" $[13]$ to assess the stuttering severity and proved to have high validity and reliability measures.

\section{Statistical analysis:}

The results were collected, tabulated, and analyzed using SPSS statistical package Version 15. Qualitative data were presented as numbers and corresponding percentages. For comparing the results Chi-Square test was used for comparison between 2 or more variables, Monte Carlo test as correction for Chi-Square test when more than $25 \%$ of cells have count less than 5 in tables $(>2 * 2)$ and Fischer Exact test was used as correction for Chi-Square test when more than $25 \%$ of cells have count less than 5 in $2 * 2$ tables .The non-parametric Spearman's rho and parametric Pearson correlation tests were used to measure the relationship between variables. $p$ value was considered statistically significant if $<0.05$. 


\section{RESULTS:}

In this study two groups were compared, the study group: children who stutter (CWS) (CWS) included 52 children, they were 43 males $(42.7 \%)$ and 9 females $(17.3 \%)$ with a mean age of $4.28 \pm 1.10$ and a range of 3 years to 7 years. The control group (CWNS) included 52 children who not stutter, they were 39 males $(75 \%)$ and 13 females $(25 \%)$ with a mean age of $4.43 \pm 1.35$ and a range of 2 years to 7 years. Non- statistical significant differences were obtained between the study group (CWS) and control group (CWNS) in terms of age and sex (Table 1)

Highly statistical significant difference were observed between the two groups as regards to the receptive language score, expressive language score, total language score, stuttering severity index and the articulatory disorders on bases of articulation test $(p<0.001)$ (Table 2$)$.

Highly statistical significant differences were obtained between the two groups as regarding DLD $(p<0.001)$; in the study group, $14(26.9 \%)$ children were diagnosed as DLD-specific language impairment (SLI) in comparison to $11(21.2 \%)$ children in the control group. In the study group, 26 (50\%) children were diagnosed as DLD-below average mentality in comparison to $5(9.6 \%)$ children in the control group. In the control group, $1(1.9 \%)$ child with DLD-MR while no child with DLD- MR in the study group and $1(1.9 \%)$ child with SSD in the control group and no child with SSD in the study group (Table 3 ).

Results of study revealed highly statistically significant positive correlation between IPD and DLD $(\mathrm{SLI})(P<0.001)$, statistically significant positive correlation between IPD and DLD (below average) $(P<0.05)$, statistically significant negative correlation between repetition of syllable and words and DLD (below average) $(P<0.05)$ and finally statistically significant positive correlation between prolongation and words and DLD (SLI) $(P<0.05)$. In addition a highly statistical significant negative correlation between IPD, repetition of syllable and words in terms of expressive language score $(P<0.001)$ and statistically significant negative correlation between IPD, repetition of syllable and words and total language score $(P<0.05)$. Finally, the results of study revealed statistically significant positive correlation between SSI and articulation among study group $(P<0.05)$ (Tables $4,5,6,7)$.

Table 1: Distribution of children in the study (104) according to demographic data.

\begin{tabular}{ccccc}
\hline & Data & & Study N=52 & Controls N=52 \\
\hline \multirow{2}{*}{ Age } & Range Mean \pm SD & $3-7$ & $2-7$ & $4.43 \pm 1.35$ \\
& & $4.28 \pm 1.10$ & 0.1 & $39(75 \%)$ \\
Sex & Male & $43(42.7 \%)$ & $13(25 \%)$ & 0.3 \\
\hline
\end{tabular}

Chi square test and Fisher's exact test for qualitative data between the two groups

$P$ value $<0.05$ (significant), $P$ value $>0.05$ (no significant), $P$ value $<0.001$ (highly significant)

Table 2: Comparison between the study group and the control group as regard the language test results, stuttering severity index and Articulation test.

\begin{tabular}{|c|c|c|c|c|}
\hline Data & & Study $\mathrm{N}=52$ & Controls $\mathrm{N}=52$ & $P$ \\
\hline Receptive language score & $\begin{array}{c}\text { Range } \\
\text { Mean } \pm \text { SD }\end{array}$ & $\begin{array}{c}50-199 \\
65.77 \pm 24.86\end{array}$ & $\begin{array}{c}50-112 \\
81.52 \pm 20.10\end{array}$ & $<0.001^{* *}$ \\
\hline Expressive language score & $\begin{array}{c}\text { Range } \\
\text { Mean } \pm \text { SD }\end{array}$ & $\begin{array}{c}46-134 \\
66.44 \pm 23.26\end{array}$ & $\begin{array}{c}46-112 \\
77.81 \pm 20.89\end{array}$ & $0.01^{* *}$ \\
\hline Total language score & $\begin{array}{c}\text { Range } \\
\text { Mean } \pm \text { SD }\end{array}$ & $\begin{array}{c}50-129 \\
63.44 \pm 20.42\end{array}$ & $\begin{array}{c}50-119 \\
80.21 \pm 21.18\end{array}$ & $<0.001^{* *}$ \\
\hline Stuttering severity index & $\begin{array}{c}\text { Range } \\
\text { Mean } \pm \text { SD }\end{array}$ & $\begin{array}{c}13-28 \\
20.31 \pm 3.55\end{array}$ & $\begin{array}{c}0-5 \\
2.40 \pm 1.83\end{array}$ & $<0.001^{* *}$ \\
\hline Articulation test & $\begin{array}{c}\text { Intact articulation } \\
\text { Speech sound disorders }\end{array}$ & $\begin{array}{l}16(30.8 \%) \\
36(69.2 \%)\end{array}$ & $\begin{array}{l}37(71.2 \%) \\
15(28.8 \%)\end{array}$ & $<0.001^{* *}$ \\
\hline
\end{tabular}

* Chi square test and Fisher's exact test for qualitative data between the two groups

$P$ value $<0.05$ (significant), $P$ value $>0.05$ (no significant), $P$ value $<0.001$ (highly significant) 
Table 3: Comparison between stuttering group and control group regarding types of language disorders.

\begin{tabular}{ccc}
\hline Data & Study N=52 & Controls N=52 \\
\hline Normal language & $12(23.1 \%)$ & $34(65.4 \%)$ \\
DLD (SLI) & $14(26.9 \%)$ & $11(21.2 \%)$ \\
DLD (below average) & $26(50 \%)$ & $5(9.6 \%)$ \\
DLD (MR) & 0 & $1(1.9 \%)$ \\
SSD & 0 & $1(1.9 \%)$ \\
\hline
\end{tabular}

$\mathrm{SLI}=$ Specific Language Impairment. DLD=Delayed Language Development. MR= Mental Retardation. SSD=Speech Sound Disorder.

Chi square test and Fisher's exact test for qualitative data between the two groups

$P$ value $<0.05$ (significant), $P$ value $>0.05$ (no significant), $P$ value $<0.001$ (highly significant)

Table 4: Correlation between SSI and (receptive language score, expressive language score and total language score) in stuttering group:

\begin{tabular}{cccc}
\hline Pearson correlation & Receptive language score & Expressive language score & Total language score \\
\hline r(SSI) & 0.326 & -0.224 & -0.350 \\
$P$-value $(\mathrm{SSI})$ & $0.001^{*}$ & $0.02^{*}$ & $<0.001^{*}$ \\
\hline
\end{tabular}

Pearson's correlation: $\quad$ P value $<0.05$ (significant)

Table 5: Correlation between core behavior and type of DLD in study group.

\begin{tabular}{ccccc}
\hline Spearman correlation & & DLD (SLI) N=14 & \multicolumn{2}{c}{ DLD (Below average) N=26 } \\
\hline IPD & $\mathrm{R}$ & $P$ & $\mathrm{R}$ & 0.316 \\
Repetition & 0.480 & $<0.001^{*}$ & -0.326 & $0.02^{*}$ \\
Prolongation & 0.198 & 0.1 & 0.213 & $0.01^{* *}$ \\
Block & 0.342 & $0.01^{*}$ & 0.1 & 0.326 \\
\hline
\end{tabular}

Pearson's correlation: $\quad$ P value $<0.05$ (significant)

Table 6: Correlation between core behaviors and(receptive language score, expressive language score and total language score) among study group:

\begin{tabular}{|c|c|c|c|c|c|c|}
\hline \multirow[t]{2}{*}{ Spearman correlation } & \multicolumn{2}{|c|}{ receptive language score } & \multicolumn{2}{|c|}{ expressive language score } & \multicolumn{2}{|c|}{ total language score } \\
\hline & $\mathrm{R}$ & $\mathrm{P}$ & $\mathrm{R}$ & $\mathrm{P}$ & $\mathrm{Nkr}$ & $\mathrm{P}$ \\
\hline IPD & 0.060 & 0.6 & -0.597 & $<0.001^{*}$ & -0.320 & $0.02^{*}$ \\
\hline Repetition & 0.088 & 0.5 & -0.494 & $<0.001^{*}$ & -0.466 & $0.001^{* *}$ \\
\hline Prolongation & -0.100 & 0.4 & -0.290 & $0.03^{*}$ & 0.066 & 0.6 \\
\hline Block & 0.022 & 0.8 & -0.256 & 0.06 & -0.186 & 0.1 \\
\hline
\end{tabular}

Table 7: Correlation between SSI and Articulatory error among study group:

\begin{tabular}{ccc} 
& Spearman Correlation & Articulatory errors \\
\hline SSI & r & 0.319 \\
$0.001^{* *}$ & $P$-value & 0.00 \\
\hline
\end{tabular}

Pearson's correlation: $\quad$ P value $<0.05$ (significant) 


\section{DISCUSSION}

Stuttering is a multifactorial speech disorder defined by frequent prolongations, repetitions, or blocks of spoken sounds and/or syllables ${ }^{[3]}$. Stuttering speech patterns are often easily identifiable; when a child is learning to talk, repetition of sounds or words, prolonged pauses, or excessively long sounds in words usually occur. Secondary behaviors (e.g., eye blinking, jaw jerking, involuntary head or other movements) that accompany stuttering can further embarrass the child, leading to a fear of speaking ${ }^{[15]}$. This study aimed at investigating the baseline data about the size and distribution of language disorders among Egyptian stuttering children (CWS). Among CWS, males represented $(77.5 \%)$ of the subjects, while females represented $(22.5 \%)$. The male to female ratio was $5: 1$. This result came in agreement with many studies $^{[16,17]}$. There was high percentage of articulation disorders among cases. This result could be explained by overlapping between the age of stuttering onset (between 2 and 4 years of age) with the period when a child is developing very rapidly in phonology and language that affect the development of speech and language during this period. This is in agreement with Wolk et al. ${ }^{[18]}$ who reported poor phonological development in stuttering children and $30 \%-40 \%$ of children who stutter have a co-occurring phonological disorder. The results obtained from the Arabic Preschool Language Scale-4 "APLS-4" revealed a high percentage among the sample complaining of low receptive, expressive, and total standard scores. These deficits in the language in stuttering children could be explained by the onset of stuttering usually occurs during a time period (2-7 years old), which coincides with the critical period of accelerated expansion in children's expressive and receptive language. Also, children in this period may attempt to simplify verbal responses as a means of coping with their stuttering. We could predict that when the syntactic demands on a language-learning child are increased, the ability to maintain fluency becomes difficult. This result was in agreement with Aneja ${ }^{[19]}$, who reported that stuttering occurs more frequently in children with delayed language disorder (DLD). A statistically significant negative correlation between SSI and standard of receptive, standard of expressive, and standard of total scores were obtained in the study group. These results excluded other causes of DLD e.g., jaundice, cyanosis, incubation, and low birth weight. We could predict that more complex language tasks productions may be accompanied by more stuttering, and children who stutter occasionally have a lower score on language tests. Also, non-fluencies may occur when children are pressed to produce utterances beyond their linguistic capacity. Statistically significant positive correlation between SSI and articulatory errors was noticed, this result was in agreement with Wolk et al. ${ }^{[24]}$. As the stutterer children with disordered phonology produced a greater percentage of dysfluent words produced per minute during a fluency interview than those who were stuttering with normal phonology. A highly statistically significant negative correlation between IPD and receptive, expressive and total language score, this could be explained as when a stuttering child is excited, or under some pressure subsequently, stuttering becomes, and the child may make underestimation of language abilities (e.g., expressive language) in these children. They may attempt to simplify verbal responses as a means of coping with their stuttering.

\section{CONCLUSION}

Determination of the relationship between language disorders and stuttering is essential because there might be a subgroup of children who require a different assessment and treatment procedures than those who only stutter, linguistic factors have been considered relevant to stuttering.

\section{RECOMMENDATION}

This work demonstrated their strong influence on the occurrence of stuttering events, or moments of stuttering in specific locations of the speech stream (e.g., the beginning of sentences and phrases) and in words of certain grammatical classes. This occurs typically in the Egyptian preschool children between age 2-7 years, which coincides with the critical period of accelerated expansion in children's expressive and receptive language.

\section{CONFLICT OF INTEREST}

There are no conflicts of interest.

\section{REFERENCES}

1. Arndt $\mathrm{J}$ and Healy E C. Concomitant Disorders in School-Age Children Who Stutter. Lang Speech Hear Serv Sch: 2001: 1;32(2):68-78.

2. Starkweather C W. Fluency and stuttering: PrenticeHall, Inc, 1987.

3. Starkweather C. Therapy for younger children. In Curlee, R. and Siegel, G. (eds.), Nature and treatment of stuttering: New directions. 1997: 257-279.

4. Jiang J, Lu C, Peng D, Zhuand C, Howell P. Classification of types of stuttering symptoms based on brain activity. PloS one. 2012; 7(6):739-747. 
5. Maguire G A, Yehand C. Ito BS. Overview of the diagnosis and treatment of stuttering. Journal of Experimental \& Clinical Medicine. 2012; 4(2): 92-97.

6. Clark HH, Tree JEF. Using uh and um in spontaneous speaking. Cognition. 2002; 84(1): 73-111.

7. Packman A, Attanasio JS. (Theoretical issues in stuttering: Taylor \& Francis, 2017.

8. Watkins R, Yairiand E, Ambrose N. Language abilities of young children who stutter. Early childhood stuttering: For clinicians by clinicians. 2005: 235-252.

9. Arndt J, Healey EC. Concomitant disorders in schoolage children who stutter. Language, speech, and hearing services in schools. 2001; 32(2):68-78.

10. Choi D, Conture EG, Tumanova V, Clark CE, Walden TA, Jones RM. Young children's family history of stuttering and their articulation, language and attentional abilities: An exploratory study. Journal of communication disorders. 2018; 71: 22-36.

11. Hanoura M, Abdel-Hamid M. Stanford Binet Intelligence test: Arabic Version. Cairo: Anglo press, 2002.

12. El-Sady S, El-Shoubary A, Hafezand G, Mohammed A. Translate, modified and standardized of Preschool Language Scale [Unpublished Thesis]: Ain Shams Medical School, 2011.

13. Abou-Elsaad T, Baz H and El-Banna M. Developing an articulation test for Arabic-speaking school-age children. Folia Phoniatrica et Logopaedica. 2009; 61(5): 275-282.
14. Rifaie N. Arabicizing and standardizing the stuttering severity instrument on the Arabic environment. Ain Shams Med J. 1999; 50: 907-914.

15. Parker JN. The Official Patient's Sourcebook on Dysphagia: A Revised and Updated Directory for the Internet Age: Icon Group Intl Inc, 2002.

16. Youssef M. Features of Arabic language as they apply to the use of co-articulation in stuttering therapy, 1986.

17. Craig A, Hancock K, Tran Y, Craigand M, Peters K Epidemiology of stuttering in the community across the entire life span. Journal of Speech, Language, and Hearing Research. 2002; 45(6): 1097-1105.

18. Wolk L, Blomgrenand M, Smith AB. The frequency of simultaneous disfluency and phonological errors in children: A preliminary investigation. Journal of fluency disorders.2000; 25(4): 269-281

19. Aneja S. Evaluation of a child with communication disorder. Indian pediatrics. 1999; 36: 891-900.

20. Wolk L, Edwards ML, Conture EG. Coexistence of stuttering and disordered phonology in young children. Journal of Speech, Language, and Hearing Research. 1993; 36(5): 906-917., Etc.[With Illustrations.]: Spastics Society Medical Education \& Information Unit, 1964. 